20

\title{
Динамика оптических и физиологических свойств кожи человека in vivo в процессе ее компрессии
}

\author{
() О.А. Зюрюкина, Ю.П. Синичкин \\ Саратовский государственный университет, \\ 410012 Саратов, Россия \\ e-mail: yusin49@gmail.com
}

Поступила в Редакцию 04.11.2018 г.

В окончательной редакции 08.02.2019 г.

Принята к публикации 23.04.2019 г.

\begin{abstract}
На основе анализа временных изменений в спектрах диффузного отражения кожи человека in vivo в диапазоне 400-2000 nm представлены результаты исследования динамики оптических и физиологических свойств кожи при наложении и снятии внешней механической компрессии. В области спектра $500-600 \mathrm{~nm}$ (области поглощения гемоглобина) временная динамика коэффициентов отражения кожи показывает, что компрессия кожи приводит к экспоненциальному уменьшению содержания в ней крови, при этом среднее время вытеснения крови из области компрессии составляет величину порядка 4-5 min. После снятия компрессии кровенаполненность кожи восстанавливается по экспоненциальному закону примерно за $30 \mathrm{~s}$. В области спектра 700-2000 nm особенностью поведения коэффициентов отражения кожи после наложения компрессии является их монотонное уменьшение по двухэкспоненциальному закону с характерными временами порядка $10 \mathrm{~s}$ и нескольких минут, которые могут быть обусловлены вытеснением свободной и связанной воды из области компрессии.
\end{abstract}

Ключевые слова: диффузное отражение кожи, дегидратация, биоткань.

DOI: $10.21883 /$ OS.2019.09.48209.329-18

\section{Введение}

К настоящему времени появился довольно большой объем публикаций, связанных с исследованиями влияния внешней механической компрессии биотканей на их оптические (поглощение и рассеяние) и физиологические (содержание крови и степень ее насыщения кислородом, содержание воды) свойства. Прикладываемая к биотканям как в условиях in vitro $[1-4]$, так и in vivo [5-16] внешняя компрессия приводит к изменению коллимированного и диффузного пропускания (in vitro) и диффузного отражения (in vitro, in vivo): она уменьшает диффузное отражения и увеличивает пропускание образцов биоткани независимо от их состояния (in vitro или in vivo), что является результатом изменения геометрии ткани (толщины и плотности) и ее оптических параметров.

Первые исследования проводились на образцах биотканей ex vivo. В работах [2,3] проведенные исследования влияния компрессии на образцы мягких тканей (склеру и аорту быка, склеру свиньи) продемонстрировали уменьшение коэффициента диффузного отражения и увеличение пропускания и коэффициентов поглощения и рассеяния в области спектра 400-1800 nm, при этом эффекты давления на оптические свойства биоткани увеличивались с увеличением давления. Были сделаны выводы о том, что компрессия увеличивает коэффициенты поглощения и рассеяния излучения в биотканях, а возможным механизмом эффекта является увеличение объемной плотности рассеивателей. Исследования диффузного отражения света и его пропускания образцами кожи свиньи ex vivo в условиях механической компрессии показали, что просветление образцов ткани в условиях компрессии улучшает визуализацию располагаемого за образцом биоткани объекта, увеличивая разрешение и контраст его изображения $[1,4]$, причем для объяснения полученных результатов предлагается учитывать динамические эффекты.

В видимом диапазоне спектра увеличение компрессии приводит к разному поведению спектров диффузного отражения: в одном случае коэффициент отражения уменьшался во всем диапазоне спектра от 400 до $2000 \mathrm{~nm}$, в другом - спектры показывали наличие изобестической точки около $600 \mathrm{~nm}$, ниже которой коэффициенты отражения увеличивались, а выше - уменьшались $[6,10]$.

В условиях in vivo изменение компрессии на поверхность биоткани обусловлено использованием волоконнооптического датчика [7,12-13]. Увеличение давления на поверхность кожи уменьшает диффузное отражение кожи в ближней инфракрасной (ИК) области спектра. При большом давлении происходят значительные спектральные изменения, при этом если компрессия достаточно продолжительна, то давление датчика на поверхность кожи влияет не только на ее оптические, но и на физиологические параметры.

Вызванные давлением спектральные изменения сильно зависят от местоположения участка биоткани и от свойств лежащих на глубине тканей. Авторами работы [7] исследовалось влияние статического и динамического (меняющегося) давления на диффузное отражение 
мягких тканей (in vitro жира и мышц и in vivo кожи разных участков ладони и запястья - над мышцей, веной и костью). В случае образцов кожи прикладываемое давление приводит к уменьшению диффузного отражения и рассеяния, при этом отмечается увеличение концентрации содержащихся в коже хромофоров (вода, гемоглобин, липиды) и соответственно увеличение поглощения.

Изменения в спектрах диффузного отражения специфичны для кожи разной морфологии в зависимости от подтканевой субстанции [11]. При наложении компрессии на кожу человека (шеи, пальца и предплечья) отмечается увеличение концентрации гемоглобина в коже шеи и его уменьшение в коже пальца и предплечья, при этом концентрация гемоглобина в коже шеи начинает уменьшаться при достаточно большом давлении (около $\left.0.9 \cdot 10^{5} \mathrm{~Pa}\right)$, в отличие от кожи пальца и предплечья, где концентрация гемоглобина уменьшается уже при малом давлении. С увеличением давления уменьшается не только содержание гемоглобина, но и степень насыщения гемоглобина кислородом. По прошествии времени порядка $1 \mathrm{~min}$ гемоглобин в коже полностью отсутствует. По сравнению с поглощением приведенный коэффициент рассеяния меняется в меньшей степени. С увеличением давления он увеличивается для кожи предплечья и уменьшается для кожи шеи и мало меняется для кожи пальца. Авторы высказывают гипотезу, что уменьшение коэффициента рассеяния при увеличении давления на кожу шеи обусловлено вытеснением воды из эпидермиса и дермы.

Эффекты влияния давления волоконно-оптического датчика на физиологические характеристики мышечной ткани мыши in vivo исследовались авторами работы [8]. Получено, что при увеличении давления содержание крови и степень оксигенации гемоглобина крови уменьшаются, в то время как приведенный коэффициент рассеяния на длине волны $700 \mathrm{~nm}$ увеличивается. Авторы предполагают, что давление датчика может увеличить плотность рассеивателей в единице объема, в результате увеличивается приведенный коэффициент рассеяния. Нужно отметить, что реакция приведенного коэффициента рассеяния на внешнее давление специфична, позволяет отличить мышечную ткань от кожи. Большой вклад в спектральные изменения диффузного отражения могут дать изменения физиологических параметров ткани, таких как изменение объема крови, оксигенация гемоглобина крови и метаболизм ткани, высказывают также авторы работы [9].

Влияние давления волоконно-оптического датчика на спектральные измерения диффузно отраженного света от поверхности внутренней стороны нижней губы человека in vivo исследовалось авторами работы [5]. Объект зондировался линейно поляризованным светом в пределах 100-200 $\mu$ m глубины приповерхностного слоя. Измерялись разностный поляризационный спектр (при средней глубине зондирования $100 \mu \mathrm{m})$ и кросс-поляризованный компонент (средняя глубина зондирования
$200 \mu \mathrm{m})$. По данным измерения рассчитывались несколько параметров, включая содержание гемоглобина, насыщение крови кислородом, диаметры кровеносных сосудов и общую интенсивность рассеяния. Получено, что при наложении компрессии значительно увеличивается разностный сигнал и значительно уменьшается кроссполяризованный компонент. Отмечено уменьшение содержания гемоглобина и степени оксигенации гемоглобина при наложении давления.

Основными факторами, влияющими на оптические и физиологические свойства биотканей в условиях компрессии, являются изменение содержания в них воды и ее перемещение в ткани, зависящее от соотношения свободной и связанной воды, а также крови, содержащейся в кровеносных сосудах подвергаемой компрессии биоткани.

Мягкие ткани являются сложными гетерогенными материалами, состоящими из воды, белков, липидов и клеток и их органелл. В частности, кожа на $70 \%$ состоит из воды (в свободном и связанном состояниях), при этом имеет место естественный градиент содержания воды в зависимости от глубины [18].

Сложная структура биотканей и связанные с этим вариации в показателе преломления их компонентов делают такие среды сильно рассеивающими в видимой и ближней ИК областях спектра. Внешняя компрессия уменьшает рассеяние излучения в тканях, поэтому данный метод является методом просветления биотканей альтернативным широко используемому методу, основанному на внедрении в биоткань химических агентов [19]. Механическое оптическое просветление достигается за счет местного сжатия ткани и предположительно объясняется смещением межклеточной воды и крови, что приводит к местной дегидратации ткани и изменению рассеивающих и поглощающих свойств ткани [20-22]. Сжатие ткани уменьшает объемную долю воды и, следовательно, уменьшает различие между показателями преломления составляющих компонентов ткани и снижает коэффициент рассеивания [5].

Для анализа структурных и оптических изменений, происходящих в кожной ткани в условиях in vivo, может быть рассмотрена двухфазная модель кожной ткани, согласно которой кожная ткань состоит из твердой эластичной матрицы, образованной коллагеновыми волокнами, и межклеточной жидкости, основную массу которой составляет вода [23]. Эта модель наилучшим образом представляет состав и механические свойства кожи. Решающим фактором в нелинейном механическом отклике кожи на ее компрессию является подвижность воды в пределах твердой матрицы, состоящей из эластичных волокон и клеток, которая определяется ее состоянием. Деформация матрицы и перемещение воды при наложении компрессии определяется соотношением свободной и связанной воды.

Достаточно подробный анализ возможных механизмов изменений, происходящих в коже свиньи in vitro и коже человека in vivo при наложении внешней компрессии, 
проведен в работе [14], при этом акцент сделан на анализе изменения содержания воды в коже в условиях компрессии. Показано, что в случае кожи свиньи in vitro наложение компрессии ведет к вытеснению свободной воды из области компрессии, в то время как перемещение связанной воды затруднено. При давлении около $400 \mathrm{kPa}$ содержание связанной воды уменьшается на $30 \%$, что, по мнению авторов, связано с деформацией ткани. Показано, что временные изменения содержания свободной и связанной воды в коже свиньи ex vivo происходят в течение времени порядка 6 min после наложения компрессии, после чего процесс стабилизируется, при этом время стабилизации зависело от величины прикладываемого давления.

Компрессия кожи человека in vivo приводит первоначально к уменьшению объема свободной воды, а при давлениях свыше $250 \mathrm{kPa}$ объем свободной воды увеличивается. Поведение связанной воды носит противоположный характер. Эксперименты показывают, что по мере уменьшения количества свободной воды в области компрессии часть связанной воды становится свободной. По сравнению с кожей свиньи толщина и количество свободной воды менее чувствительны к давлению.

В работах $[15,16]$ представлены результаты исследования влияния внешней механической компрессии на спектры диффузного отражения кожи человека in vivo в диапазоне спектра 400-2000 nm. Отмечается, что процесс стабилизации спектров диффузного отражения в условиях внешней компрессии носит инерционный характер, при этом при внешней механической компрессии величиной $110 \mathrm{kPa}$ время стабилизации спектров составляет величину порядка нескольких минут, в то время как после снятия внешней компрессии кожа восстанавливает свои оптические параметры в видимой и ближней ИК областях в течение времени порядка $50 \mathrm{~min}$.

Исследование инерционности процессов временных изменений оптических и физиологических свойств кожи человека in vivo не только важно для понимания процессов, происходящих в коже под влиянием внешней компрессии, но и в связи с тем, что инерционность процессов влияет на результаты измерений, которые зависят от времени задержки между наложением компрессии и измерениями.

Частично этот вопрос изучен в работах $[15,16]$, тем не менее он остается актуальным, поэтому целью настоящей работы являлось выявление закономерностей динамики оптических и физиологических свойств кожи человека in vivo в процессе наложения и снятия внешней механической компрессии разной величины и разного размера области приложения внешнего давления.

\section{Экспериментальная установка, материалы и методы}

Приведенная на рис. 1 установка для измерения спектров диффузного отражения предплечья человека

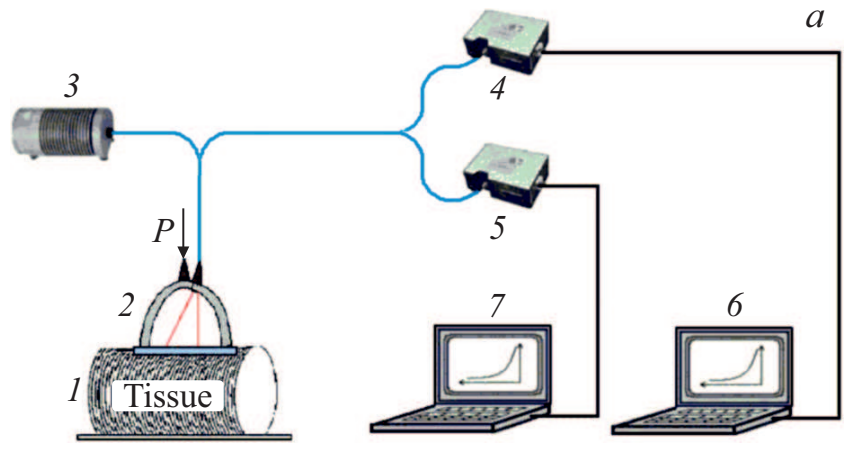

Рис. 1. Схема экспериментальной установки. 1 - объект исследования, 2 - волоконно-оптический датчик, 3 - осветительное устройство, 4,5- волоконно-оптические спектрометры, 6, 7 - персональные компьютеры.

in vivo (1) включала в себя волоконно-оптический датчик (2), осветительное устройство HL-2000 (Ocean Optics, CША) (3) и два волоконно-оптических спектрометра USB4000 (Ocean Optics, США; область регистрации спектров 400-1000 nm) и NIRQuest512-2.2 (Ocean Optics, США; область регистрации спектров 900-2200 nm) (4,5), сопряженных с персональными компьютерами $(6,7)$, и обеспечивала регистрацию спектров диффузно отраженного биотканью света в диапазоне от 400 до $2000 \mathrm{~nm}$.

Для детектирования диффузно отраженного кожей света использовались волоконно-оптический датчик собственной конструкции, подробно описанный в работах $[15,16]$, и два волоконно-оптических датчика фирмы Ocean Optics (R400-7-VIS/NIR и R600-7-VIS-125F). $\mathrm{B}$ первом случае конструкция датчика включала полукольцо радиусом $40 \mathrm{~mm}$ с закрепленными в нем двумя волоконно-оптическими световодами для подвода излучения к поверхности кожи и сбора отраженного ей света (рис. 2,a). При регистрации спектров диффузного отражения кожи в условиях ее внешней механической компрессии между полукольцом волоконно-оптического датчика и поверхностью кожи помещалось тонкое кварцевое стекло диаметром $30 \mathrm{~mm}$, на которое оказывалось давление $p$ в диапазоне от 0 до $10^{6} \mathrm{~Pa}$. Во втором случае регистрация спектров диффузного отражения кожи осуществлялась с помощью двух волоконно-оптических датчиков фирмы Ocean Optics (R400-7-VIS/NIR и R600-7-VIS-125F). Датчики содержали семь световодов диаметром 400 или $600 \mu \mathrm{m}$, шесть из которых, располагаемых по окружности, служили для подвода излучения к объекту исследования, а центральный световод обеспечивал детектирование света, диффузно отраженного биотканью. Приемный световод был состыкован с волоконно-оптическими спектрометрами USB4000 и NIRQuest512-2.2, которые, в свою очередь, были связаны с персональными компьютерами.

Датчики крепились в специальных держателях, которые обеспечивали необходимую область приложения 

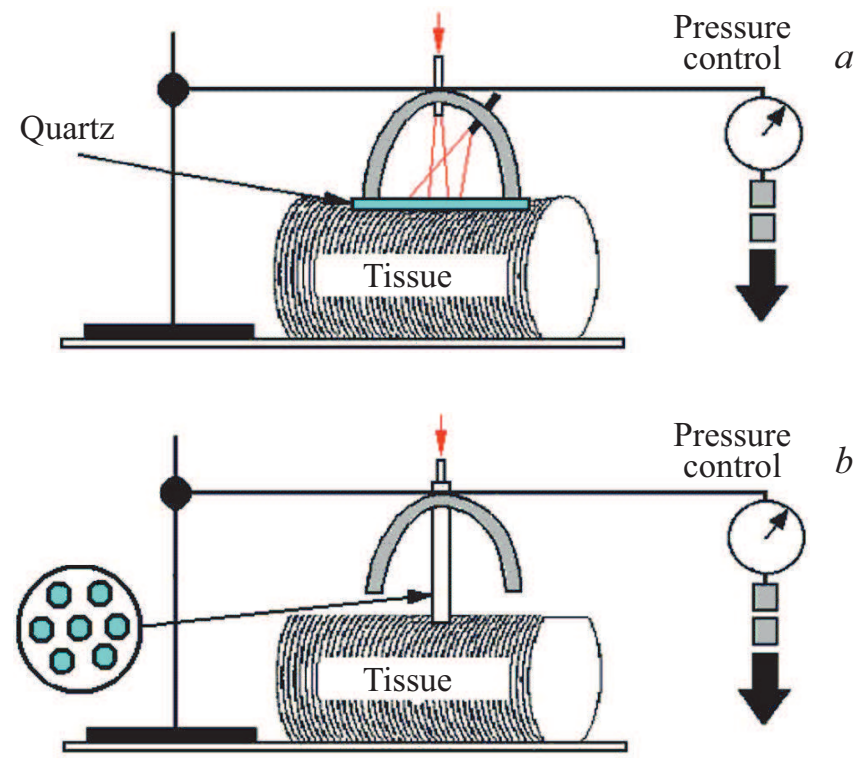

Pис. 2. Схема устройства для регистрации спектров диффузного отражения в условиях внешней механической компрессии биоткани. (a) датчик $1,(b)$ датчик 2.

давления на кожу. В свою очередь, датчики с держателями крепились на полукольце радиусом $40 \mathrm{~mm}$, на которое оказывалось внешнее давление в диапазоне от 0 до $10^{6} \mathrm{~Pa}$ (рис. $2, b$ ). Размер области прикладывания давления обеспечивали либо торцы датчиков $(3.2,6.3 \mathrm{~mm})$, либо держатели (10, 13.8 и $15.8 \mathrm{~mm})$.

Объектом исследования являлась кожа внутренней стороны предплечья человека in vivo. Измерения спектров диффузного отражения кожи человека проводились на 10 добровольцах в возрасте 20-65 лет с кожей III и IV типа, один доброволец имел кожу $\mathrm{V}$ типа по Фитцпатрику [17] .

Конструкция крепления датчиков в виде полукольца позволяла при его фиксировании вдоль предплечья минимизировать изменения в геометрии измерений, следствием чего являлась высокая степень воспроизводимости результатов (более 95\%).

\section{Результаты эксперимента и их обсуждение. Динамика содержания крови и степени оксигенации гемоглобина крови}

Временная динамика спектров диффузного отражения кожи in vivo в диапазоне спектра 500-600 nm при наложении компрессии обусловлена характером изменения рассеивающих и поглощающих свойств биоткани в условиях внешней механической компрессии [15]. Основными хромофорами кожи, определяющими спектр диффузного отражения кожи в видимом диапазоне спектра, являются пигмент меланин и гемоглобин крови, содержащейся в папиллярной дерме [24]. Очевидно, что при наложении компрессии содержание меланина в коже не меняется, в то время как содержание крови в кожной ткани может существенно изменяться, особенно при наложении на кожу больших давлений (порядка $100 \mathrm{kPa}$ ). Об этом свидетельствует поведение провала в спектре диффузного отражения кожи в спектральной области 500-600 nm, обусловленного поглощением гемоглобина.

Внешняя компрессия приводит к уменьшению коэффициента отражения кожи во всей спектральной области от 500 до $2000 \mathrm{~nm}$ (рис. 3), при этом уменьшается величина провала в области спектра 500-600 nm, который практически исчезает через $5 \mathrm{~min}$ после наложения компрессии. После снятия внешней компрессии в области спектра 500-600 nm провал вновь образуется. Временные изменения коэффициентов отражения в области 500-600 nm (области поглощения гемоглобина) оказываются чувствительны к количеству крови в коже, размеру области приложения компрессии и величине этой компрессии, при этом динамика содержания крови в коже видна из временного поведения индекса эритемы кожи в процессе ее компрессии.

Известно, что оценка содержания хромофоров в коже возможна по ее спектрам диффузного отражения путем введения индексов пигментации [24].

Индекс эритемы $E$, пропорциональный количеству содержащейся в коже крови, определяется путем сравнения величин оптической плотности кожи в спектральной области 510-610 nm [24]

$$
\begin{aligned}
E= & 100\left[\mathrm{OD}_{560}+1.5\left(\mathrm{OD}_{545}+\mathrm{OD}_{575}\right)\right. \\
& \left.-2.0\left(\mathrm{OD}_{510}+\mathrm{OD}_{610}\right)\right]
\end{aligned}
$$

где оптическая плотность $\mathrm{OD}(\lambda)$ кожи связана со спектром диффузного отражения ткани $R(\lambda)$ простым соотношением $\mathrm{OD}(\lambda)=-\log (R(\lambda))$, индексы определяют длины волн в нанометрах, на которых определялась оптическая плотность.

Степень оксигенации гемоглобина крови в биотканях, определяемая концентрациями дезоксигемоглобина, $C_{\text {deоху }}$, и оксигемоглобина, $C_{\text {oxу }}$

$$
Y=\frac{C_{\text {oxy }}}{C_{\text {oxy }}+C_{\text {deoxy }}}
$$

может быть рассчитана согласно следующему выражению [25]:

$$
Y=\alpha\left(\left(\frac{\mathrm{OD}_{570}-\mathrm{OD}_{557}}{13}-\frac{\mathrm{OD}_{557}-\mathrm{OD}_{545}}{12}\right) \frac{1}{H}+\beta\right),
$$

где коэффициенты $\alpha$ и $\beta$ определяются эмпирически: $\alpha=31, \beta=1$, а величина индекса гемоглобина $H$ (аналог индекса эритемы) определяется следующим обра3ом [25]:

$$
H=\frac{\mathrm{OD}_{545}-\mathrm{OD}_{530}}{16}-\frac{\mathrm{OD}_{570}-\mathrm{OD}_{545}}{25} .
$$


Таблица 1. Времена выдавливания крови из области компрессии для разных датчиков. Компрессия $55.5 \mathrm{kPa}$

\begin{tabular}{c|c}
\hline Датчик, $\mathrm{mm}$ & \multicolumn{1}{|c}{ Время, $\mathrm{s}$} \\
\hline 30.0 & $30.0 \pm 8.2$ \\
15.8 & $23.0 \pm 2.6$ \\
13.8 & $18.5 \pm 2.2$ \\
10.0 & $13.0 \pm 3.8$ \\
6.3 & $6.7 \pm 2.1$ \\
3.2 & $3.7 \pm 1.7$
\end{tabular}

На рис. 4 приведена динамика индекса эритемы кожи при наложении внешней компрессии и после ее снятие. Временная зависимость индекса эритемы получена с временным шагом, равным $2 \mathrm{~s}$. Наложение внешней компрессии приводит к уменьшению содержания крови в коже, причем временное изменение индекса эритемы кожи хорошо аппроксимируется экспоненциальной функцией.

По прошествии времени, определяющего транспорт крови из области компрессии, содержание оставшейся в области компрессии кожи крови перестает меняться (индекс эритемы стабилизируется). Среднее время вытеснения крови из области компрессии зависит от величины приложенного давления $(18.19 \pm 10.34 \mathrm{~s}$ при компрессии $p=41.6 \mathrm{kPa}$ до $58.16 \pm 16.6 \mathrm{~s}$ при компрессии $p=76.2 \mathrm{kPa})$. С увеличением размера области приложения давления время вытеснения крови также увеличивается. Это видно из табл. 1, где приведены средние времена вытеснения крови при использовании разных датчиков.

Количество крови, вытесненной из области компрессии, зависит от величины компрессии (рис. 5), при этом при компрессии более $110 \mathrm{kPa}$ кровь вытесняется полностью (рис. 6). При снятии внешней компрессии в течение времени около $30 \mathrm{~s}$ происходит резкое увеличение содержания крови в объеме кожной ткани, которая подвергалась компрессии, далее значения индекса эритемы возвращаются в исходное состояние. Это происходит в течение $30-50 \mathrm{~min}$ в зависимости от величины компрессии, при этом релаксация индекса эритемы хорошо описывается экспоненциальной функцией.

Динамика степени оксигенации гемоглобина в условиях механической компрессии и после ее снятия приведена на рис. 7. После наложения компрессии уменьшение содержания крови сопровождалось уменьшением степени оксигенации содержащегося в ней гемоглобина. Снятие компрессии приводило к резкому (до 2.7 раз) увеличению степени оксигенации [15].

Нужно отметить, что в спектрах диффузного отражения кожи проявляется в основном кровь, находящаяся в поверхностном сосудистом сплетении, и степень оксигенации гемоглобина является параметром, зависящим от соотношения артериальной и венозной

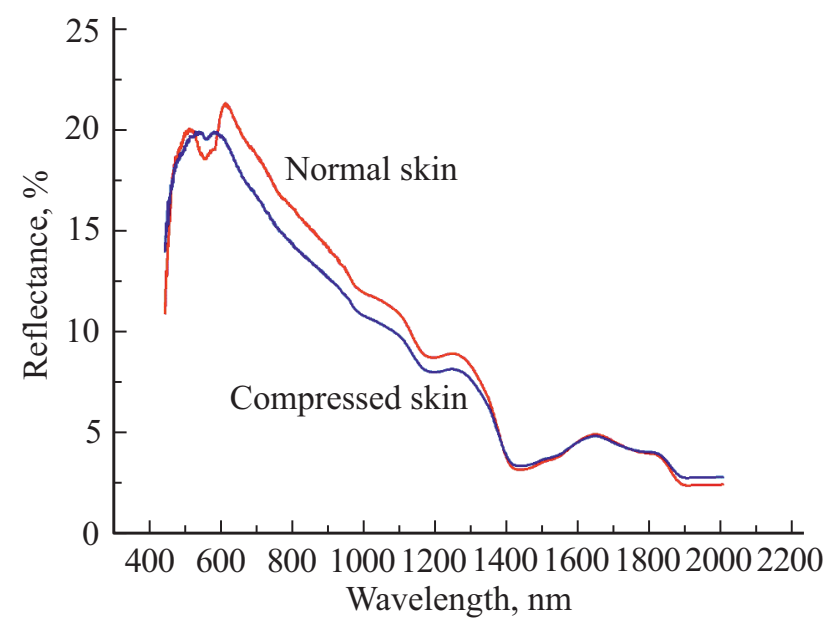

Рис. 3. Спектры диффузного отражения кожи человека in vivo. Компрессия $p=110 \mathrm{kPa}$.

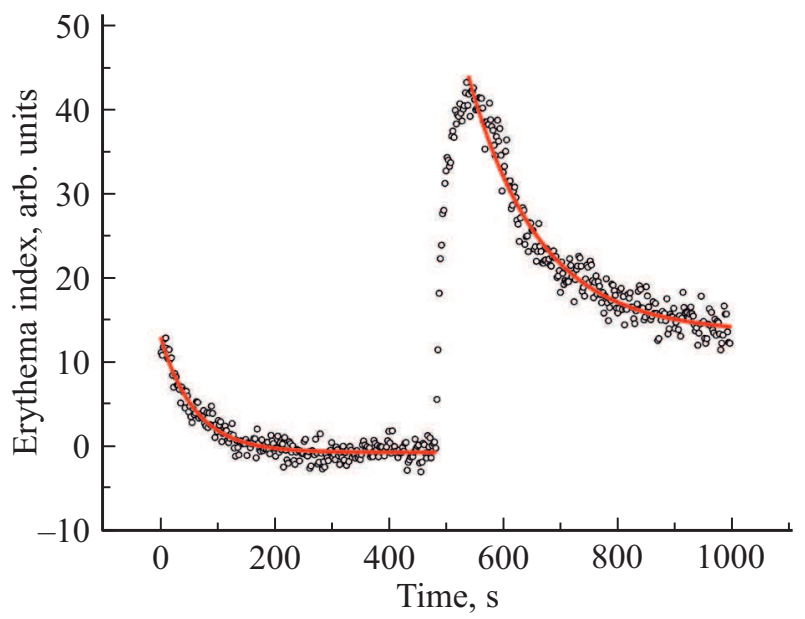

Рис. 4. Динамика индекса эритемы в условиях внешней механической компрессии (интервал времени 0-480s) и после ее снятия (интервал времени свыше $480 \mathrm{~s}$ ). Давление $200 \mathrm{kPa}$. Сплошные линии - аппроксимация экспериментальных данных экспоненциальными функциями.

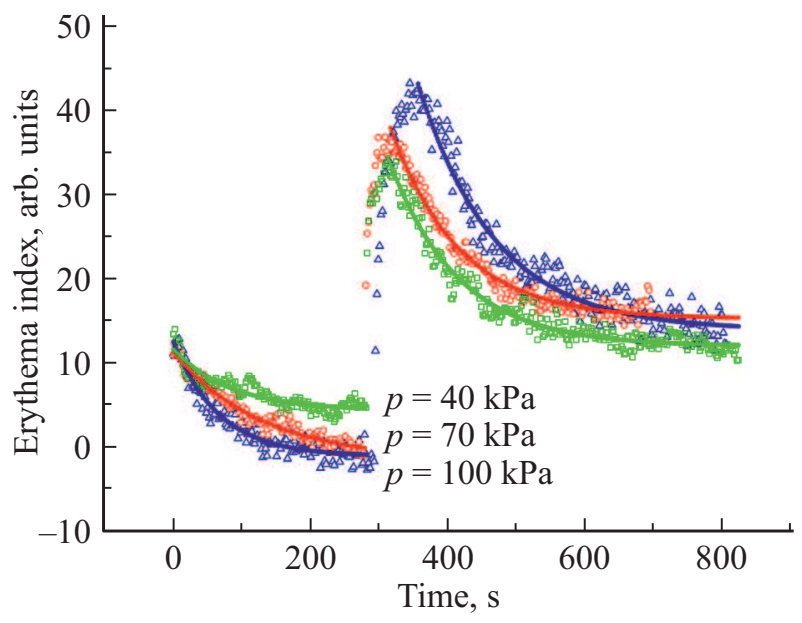

Рис. 5. Динамика индекса эритемы кожи при разной величине внешней компрессии. Линии - аппроксимация экспоненциальными функциями. 


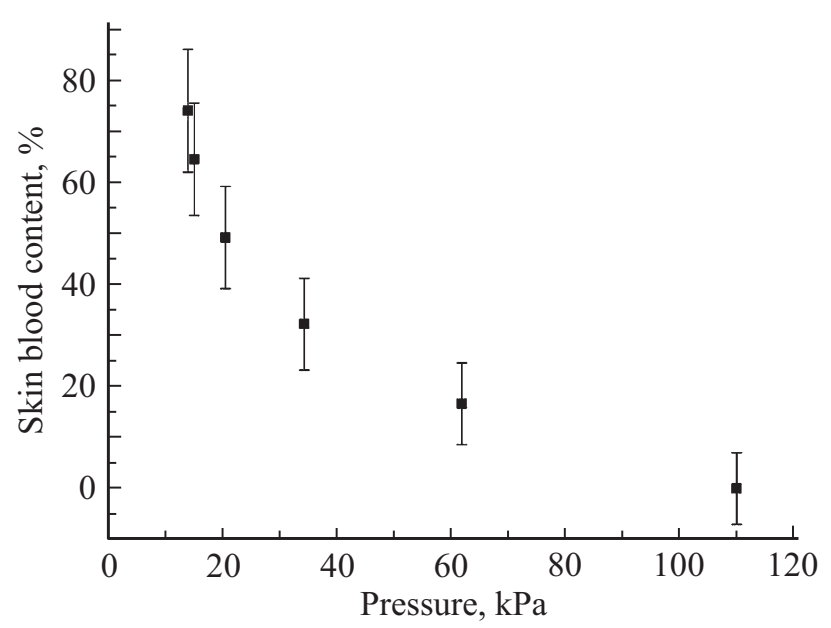

Рис. 6. Содержание крови (гемоглобина) в коже в зависимости от приложенной компрессии.

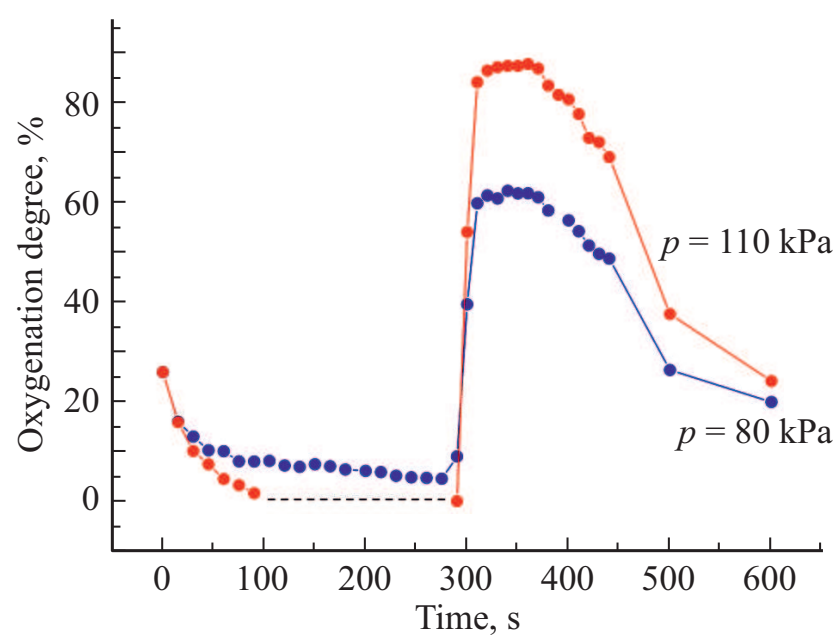

Pис. 7. Динамика степени оксигенации гемоглобина крови кожи при наложении внешней компрессии (интервал времени 0-290 s) и при ее снятии (интервал времени свыше $290 \mathrm{~s}$ ). Штриховая линия показывает временной диапазон, когда кровь в кожной ткани отсутствует.

крови в зондируемом объеме кожной ткани. Если в нормальном состоянии степень тканевой оксигенации составляла величину порядка $32 \%$, то возможной причиной увеличения степени оксигенации после снятия компрессии может быть резкий вброс артериальной крови в объем кожной ткани, который подвергался сдавливанию, так как клапаны вен не дают выдавленной венозной крови вернуться в область компрессии после ее снятия [15].

\section{Динамика дегидратации кожи в процессе ее компрессии}

В спектральном диапазоне 600-2000 nm на спектр диффузного отражения кожи в условиях наложения и снятия компрессии существенное влияние оказывает количество содержащейся в коже воды, при этом в области спектра 600-800 nm содержание воды определяет рассеивающие свойства кожи, а в диапазоне спектра свыше $800 \mathrm{~nm}$ помимо рассеивающих свойств содержание воды определяет и поглощающие свойства кожи.

На рис. 8 приведена динамика коэффициента отражения кожи на длине волны $800 \mathrm{~nm}$ при наложении и снятии компрессии. Отличительной особенностью поведения коэффициента отражения после наложения компрессии является его монотонное уменьшение по двухэкспоненциальному закону. Характерные времена затухания экспоненциальных функций составляют величины порядка нескольких секунд (быстрая релаксация) и нескольких минут (медленная релаксация). В табл. 2 приведены времена быстрой и медленной релаксации при разных величинах компрессии, усредненные по коэффициентам отражения на разных длинах волн.

Двухэкспоненциальная временная зависимость коэффициентов отражения кожи при наложении компрессии может быть обусловлена наличием в коже как свободной, так и связанной воды. В первоначальный момент приложения давления происходит резкая деформация и сжатие коллагеновой матрицы кожи (согласно двухфазной модели), которое сопровождается удалением из области компрессии свободной воды (быстрая релаксация). В дальнейшем скорость вытекания воды из области кожной ткани, подверженной компрессии, уменьшается и происходит процесс медленной дегидратации этого объема кожи (медленная релаксация), в который вовлекается связанная вода.

Можно провести аналогию между процессом дегидратации образцов кожи in vitro под действием осмотических оптических просветляющих агентов [26] и кожи in vivo в процессе ее сжатия под действием компрессии.

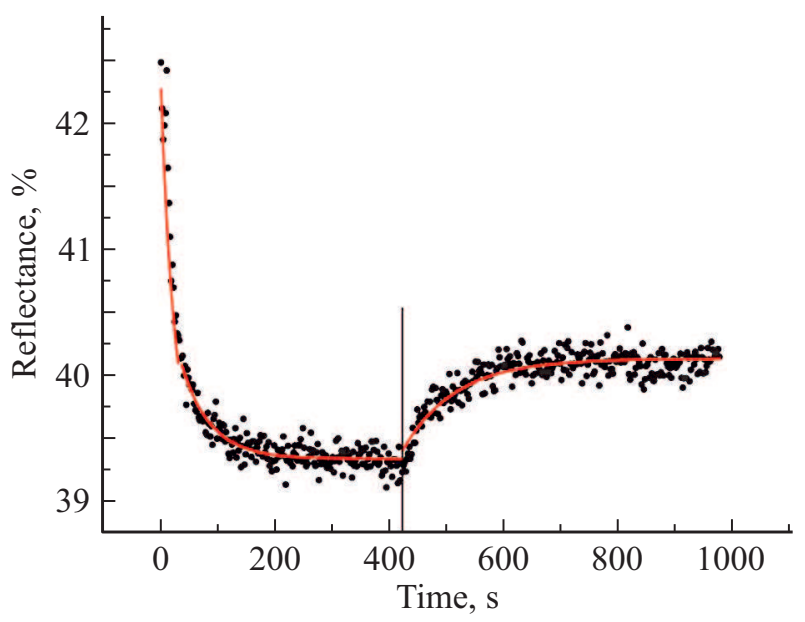

Рис. 8. Динамика коэффициента отражения кожи на длине волны $800 \mathrm{~nm}$ в процессе наложения (временной интервал $0-420$ s) и снятия (временной интервал свыше 420 s) внешней компрессии. $p=100 \mathrm{kPa}$. Сплошные кривые - аппроксимация экспоненциальными функциями. Вертикальная линия - время снятия компрессии. 
Таблица 2. Времена быстрой и медленной релаксации для усредненных по длинам волн коэффициентов отражения при разных величинах компрессии

\begin{tabular}{c|c|c}
\hline Компрессия, $\mathrm{kPa}$ & Быстрая релаксация, $\mathrm{s}$ & Медленная релаксация, $\mathrm{s}$ \\
\hline 27.7 & $12.72 \pm 6.20$ & $160.93 \pm 96.51$ \\
41.6 & $8.28 \pm 3.83$ & $105.56 \pm 44.68$ \\
55.5 & $5.18 \pm 1.16$ & $82.50 \pm 46.50$
\end{tabular}

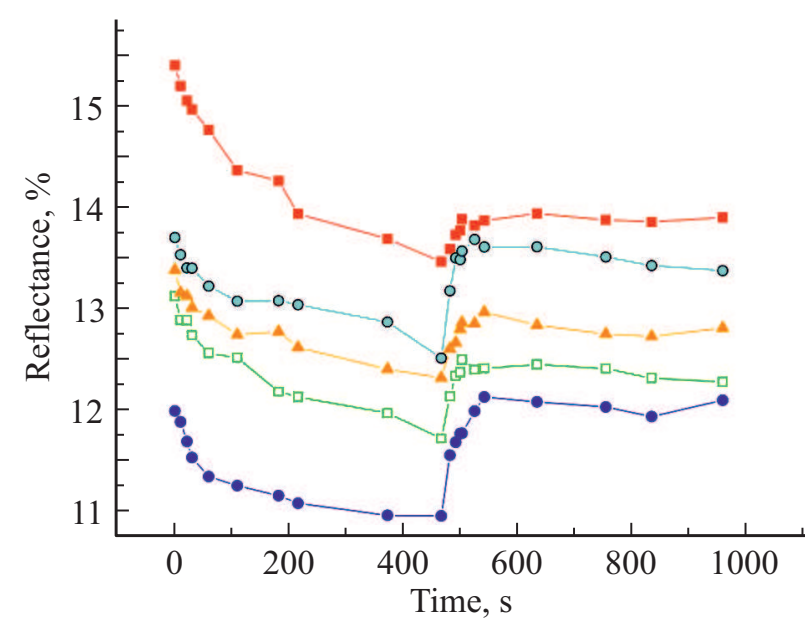

Рис. 9. Временные изменения коэффициента отражения кожи в процессе наложения компрессии (интервал времени $0-460 \mathrm{~s}$ ) и после ее снятия (интервал времени свыше $460 \mathrm{~s}$ ). Разные добровольцы.

В обоих случаях происходит удаление воды из кожи по экспоненциальному закону. Следуя авторам работы [26], можно временную зависимость степени дегидратации кожи записать в следующем виде:

$$
H_{D}(t)=A_{D}\left(1-\exp \left(-t / \tau_{D}\right)\right)
$$

где $A_{D}$ - безразмерный параметр, характеризует максимальную степень дегидратации, $\tau_{D}$ - постоянная времени дегидратации, характеризующая скорость процесса.

Если анализ процесса дегидратации образцов кожи in vitro можно проводить на основе изменения их массы в процессе уменьшения содержания в них воды, то дегидратацию кожи in vivo при наложении компрессии можно проводить путем анализа изменений коэффициентов отражения. Характерные времена экспоненциального уменьшения коэффициентов отражения в спектральной области 600-800 nm после наложения компрессии являются не чем иным, как временами дегидратации кожи, понимая под этим ее обезвоживание. На рис. 9 приведены временные изменения коэффициента отражения кожи разных волонтеров на длине волны $800 \mathrm{~nm}$.

Как сказано выше, временные изменения коэффициентов отражения $R$ в течение времени компрессии описываются экспоненциальными функциями, отражающие процесс дегидратации кожи под действием компрессии, при этом уменьшение $R$ обусловлено уменьшением рассеяния в коже в результате дегидратации. Однако процессы дегидратации образцов кожи in vitro и кожи in vivo, несмотря на их схожесть, имеют принципиальные отличия. Основные параметры $A_{D}$ и $\tau_{D}$, описывающие процесс дегидратации кожи in vitro, для кожи in vivo недостижимы. Очевидно, что полностью обезводить кожу in vivo невозможно, поэтому теряет смысл говорить о максимальной степени дегидратации. Можно говорить только о степени дегидратации кожи в результате определенной компрессии и времени дегидратации, характеризующем скорость данного определенного процесса, а на эти величины влияют величина компрессии и размеры области ее приложения. Так, относительные уменьшения коэффициента отражения, наибольшие для датчика $6.3 \mathrm{~mm}(11.3+2.2 \%)$, имеют меньшие значения для датчиков $10 \mathrm{~mm}(9.5+2.0 \%)$ и $13.9 \mathrm{~mm}(9.5+1.7 \%)$ и наименьшие для датчика $15.8 \mathrm{~mm}(7.2+3.4 \%)$.

Весь процесс стабилизации спектров может происходить приблизительно в течение времени до $6 \mathrm{~min}$. Снятие компрессии приводит к восстановлению первоначального содержания воды в коже, при этом время данного процесса составляет величину порядка $50 \mathrm{~min}$.

\section{Заключение}

Процесс изменения спектра диффузного отражения кожи in vivo при наложении и снятии внешней механической компрессии носит инерционный характер, зависящий от динамики поглощающих и рассеивающих свойств кожи. В свою очередь, временное изменение поглощающих и рассеивающих свойств кожи зависит от динамики процессов изменения физиологических свойств кожи (содержания и степени насыщения кислородом крови и содержания воды).

В области спектра 500-600 nm спектральные изменения в диффузном отражении кожи обусловлены поглощением гемоглобина, содержащимся в коже крови. После наложения компрессии происходит экспоненциальное уменьшение индекса эритемы, свидетельствующее об уменьшении содержания крови в коже. Среднее время вытеснения крови из области компрессии увеличивается с увеличением области приложения давления приложенного давления и величины давления и составляет величину порядка 4-5 min. При давлении порядка $110 \mathrm{kPa}$ 
кровь (гемоглобин) из области компрессии удаляется полностью. После снятия компрессии в течение порядка $30 \mathrm{~s}$ резкое увеличение содержания крови в объеме кожной ткани, которая подвергалась компрессии, а затем индекс эритемы возвращается в исходное состояние в течение 30-50 min в зависимости от величины компрессии, при этом релаксация индекса эритемы хорошо описывается экспоненциальной функцией.

Поведение спектра диффузного отражения кожи в условиях компрессии в области спектра 700-800 nm определяется изменением только рассеивающих свойств кожи, которые, в свою очередь, определяются количеством содержащейся в коже воды, а в области свыше $800 \mathrm{~nm}$ содержание воды определяет и поглощение кожи. В ближней ИК области отличительной особенностью поведения коэффициента отражения после наложения компрессии является его монотонное уменьшение по двухэкспоненциальному закону с характерными временами порядка нескольких секунд (быстрая релаксация) и нескольких минут (медленная релаксация). Двухэкспоненциальный закон обусловлен, по нашему мнению, разными скоростями перемещения из области приложения компрессии свободной и связанной воды. Весь процесс стабилизации спектров может происходить приблизительно в течение времени до $6 \mathrm{~min}$. Снятие компрессии приводит к восстановлению первоначального содержания воды в коже, при этом время данного процесса составляет величину порядка $50 \mathrm{~min}$.

По аналогии с анализом процесса дегидратации образцов кожи in vitro, проводимым на основе уменьшения их массы в процессе дегидратации, дегидратацию кожи in vivo в условиях компрессии можно проводить путем анализа изменений коэффициентов отражения кожи в ближней ИК области после наложения компрессии.

Дегидратация и обескровленность биоткани, являющиеся результатом ее компрессии, позволяет говорить о компрессионном методе оптического просветления тканей как альтернативном иммерсионному методу. Компрессия биоткани приводит к ее механическому сжатию и, как следствие, к вытеснению крови и воды из области компрессии, результатом чего уменьшается и рассеяние света биотканями, и поглощение света в тех спектральных областях, где велико поглощение гемоглобина и воды. Техника оптического просветления позволяет свету глубже проникать в биоткань, улучшая тем самым качество различных диагностических и терапевтических технологий, связанных со светом. Эффекты, связанные с просветлением биоткани, дают несомненное преимущество при использовании метода оптической когерентной томографии (ОКТ). Уменьшение доли воды в коже дает существенный выигрыш в яркости ОКТ-изображения биоткани (в коже увеличение яркости составляет порядка $1.5 \mathrm{~dB}$ на глубине $1 \mathrm{~mm}$ ), при этом эффект сильнее при зондировании кожи излучением на длинах волн, где поглощение воды меньше. Увеличение яркости ведет к лучшей визуализации и увеличению контраста наблюдения микронеоднородностей на большей глубине визуализации, а также получать дополнительную информацию об морфофункциональном состоянии объекта. К примеру, в ИК области это позволяет дифференцировать воспаление и карциному прямой кишки при диагностике методом ОКТ ex vivo, а в видимой определять уровень содержания каротиноидов в дерме кожи по спектру ее отражения в области 467-515 nm при удалении из кожи крови.

\section{Соблюдение этических стандартов}

Исследования с участием добровольцев проводились с их согласия на основании Федерального закона Российской Федерации и Хельсинкской декларации о надлежащей клинической практике. Получена рекомендация комитета по этике ФГБОУ ВО „Саратовский Государственный медицинский университет им. В.И. Разумовского“ Министерства здравоохранения РФ.

\section{Конфликт интересов}

Авторы заявляют, что у них нет конфликта интересов.

\section{Список литературы}

[1] Izquierdo-Roman A., Vogt W.C., Hyacinth L., Rylander C.G. // Laser Surg. Med. 2011. V. 43. N 8. P. 814.

[2] Chan E K., Sorg B., Protsenko D. O'Neil M., Motamedi M., Welch A.J. // IEEE J. Sel. Top. Quantum Electron. 1996. V. 2. N 4. P. 943.

[3] Shangguan H., Prahl S.A., Jacques S.L., Casperson L.W., Gregory K.W. // Proc. SPIE. 1998. V. 3254. P. 366.

[4] Vogt W.C., Izquierdo-Roman A., Nichols B., Lim L., Tunnell J.W., Rylander C.G. // Laser Surg. Med. 2012. V. 44. N 4. P. 303.

[5] Ruderman S., Gomes A.J., Stoyneva V., Rogers J.D., Fought A.J., Jovanovic B.D., Backman V. // Biomed. Opt. Express. 2010. V. 1. N 2. P. 489.

[6] Синичкин Ю.П., Ути С.Р., Пилипенко Е.А. // Опт. и спектр. 1996. Т. 80. № 2. С. 260.

[7] Chen W., Liu R., Xu K., Wang R.K. // J. Phys. D: Appl. Phys. 2005. V. 38. N 15. P. 2691.

[8] Reif R., Amorosino M.S., Calabro K.W., A'Amar O., Singh K.S., Bigio I.J. // J. Biomed. Opt. 2008. V. 13. N 1. P. 010502.

[9] Ti Y., Lin W.C. // Opt. Express. 2008. V. 16. N 6. P. 4250.

[10] Delgado Atencio J.A., Orozco E.E., Vázquez Y., Montiel S., Cunill Rodríguez M., Castro Ramos J., Gutiérrez J.L., Martínez F. // Opt. Mem. Neur. Networks (Information Optics). 2009. V. 18. N 1. P. 6.

[11] Lim L., Nichols B., Rajaram N., Tunnell J.W. // J. Biomed. Opt. 2011. V. 16. N 1. 011012.

[12] Cugmas B., Bürmena M., Bregar V., Pernuša F., Likar B. // J. Biomed. Opt. 2013. V. 18. N 4. P. 047002.

[13] Cugmas B., Bürmena M., Bregar V., Pernuša F., Likar B. // J. Biomed. Opt. 2014. V. 19. N 3. P. 037002.

[14] Li C., Jiang J., Xu K. // J. Innovative Opt. Health Sci. 2013. V. 6. N 1. P. 1350005. 
[15] Нахаева И.А., Мохаммед М.Р., Зюрюкина О.А., Синичкин Ю.П. // Опт. и спектр. 2014. Т. 117. № 3. C. 522; Nakhaeva I.A., Mohammed R.M., Zyuryukina O.A., Sinichrin Yu.P. // Opt. Spectrosc. 2014. V. 117. N 3. P. 506.

[16] Нахаева И.А., Зюрюкина О.А., Мохаммед М.Р., Синичкин Ю.П. // ОПт. и спектр. 2015. Т. 118. № 5. C. 152; Nakhaeva I.A., Zyuryukina O.A., Mohammed R.M., Sinichrin Yu.P. // Opt. Spectrosc. 2015. V. 118. N 5. P. 834.

[17] Fitzpatrick T.B. // Arch Dermatol. 1988. V. 124. P. 869.

[18] Delalleau A., Josse G., Lagarde J.-M., Zahouani H., Bergheau J.-M. // Skin Res. Technol. 2008. V. 14. N 2. P. 152.

[19] Tuchin V.V. // J. Phys. D: Appl. Phys. 2005. V. 38. N 15. P. 2497.

[20] Rylander C.G., Stumpp O.F., Milner T.E., Kemp N.J., Mendenhall J.M., Diller K.R., Welch A.J. // J. Biomed. Opt. 2006. V. 11. N 4. P. 041117.

[21] Xu X., Wang R.K. // Med. Phys. 2003. V. 30. N 6. P. $1246-1253$.

[22] Gurjarpadhye A.A., Vogt W.C., Liu Ya., Rylander C.G. // Int. J. Biomed. Imag. 2011. P. 817250.

[23] Oomens C.W.J., Vancampen D.H., Grootenboer H.J. // J. Biomech. 1987. V. 20. N 9. P. 877.

[24] Синичкин Ю.П., Ути, С.P. In vivo отражательная и флуоресцентная спектроскопия кожи человека. Саратов: Изд-во Сарат. ун-та, 2001. 92 с.

[25] Hajizadeh-Saffar M., Feather J.W., Dawson J.B. // Phys. Med. Biol. 1990. V. 35. 9. P. 1301.

[26] Genina E.A., Bashkatov A.N., Korobko A.A., Zubkova E.A., Tuchin V.V., Yaroslavsky I., Altshuler G.B. // J. Biomed. Opt. 2008. V. 13. N 2. P. 021102 